Pacific Journal of Mathematics

IDEALS OF THE PRINCIPAL CLASS, $R$-SEQUENCES AND A 


\title{
IDEALS OF THE PRINCIPAL CLASS, R-SEQUENCES AND A CERTAIN MONOIDAL TRANSFORMATION
}

\author{
EDWARD D. DAVIS
}

\begin{abstract}
We consider the algebra generated over the ring $R$ by the quotients $\left\{x_{1} / x, \cdots, x_{n} / x\right\}$. This "monoidal transform" $R\left[x_{1} / x\right.$, $\left.\cdots, x_{n} / x\right]$ may be regarded as the homomorphic image of the polynomial ring $R\left[X_{1}, \cdots, X_{n}\right]$. Examination of the kernel of this homomorphism gives in one instance the theorem of analytic independence of systems of parameters and in another the analogous theorem about $R$-sequences in arbitrary commutative rings. We combine these results with some older work of ours (included in an appendix) to give several characterizations of ideals in Noetherian rings generated by $R$ sequences.
\end{abstract}

1. Notation and preliminaries. We shall interpret "ring" to mean commutative ring with unity. Given the ring $R$, consider the ideal $I=\left(x, x_{1}, \cdots, x_{n}\right)$, where $x$ is not a divisor of zero, and the ring $S=R\left[x_{1} / x, \cdots, x_{n} / x\right]$, a subring of the total quotient ring of $R$. Observe that the extension of $I$ to $S$ is principal, namely, $I S=x S$. Regard $S$ as the homomorphic image of the polynomial ring $R[X]=$ $R\left[X_{1}, \cdots, X_{n}\right]$ (substitute $x_{i} / x$ for $X_{i}$ ) and let $Q$ denote the kernel of this homomorphism. Clearly $Y_{i}=x X_{i}-x_{i} \in Q$; let $Q^{\prime}$ be the $R[X]$ ideal $\left(Y_{1}, \cdots, Y_{n}\right)$. Some simple remarks:

a. Given $f \in Q$ of degree $t$, since $x X_{i}=Y_{i}+x_{i}, x^{t} f=g\left(Y_{1}, \cdots, Y_{n}\right)$, where $g \in R[X]$ and has constant term 0 . Thus $x^{t} f \in Q^{\prime}$. It follows that $Q=Q^{\prime}$ if $Q^{\prime}$ is a prime ideal, and that $Q^{\prime}$ contains $x^{r} Q$ for sufficiently large $r$ if $Q$ is finitely generated. Suppose that $\operatorname{IR}[X]$ contains $Q$. Then since $x_{i}=x X_{i}-Y_{i}, f=x f_{1}+g_{1}$, where $g_{1} \in Q^{\prime}$ and $f_{1} \in Q$. It follows that for each $r, f=x^{r} f_{r}+g_{r}$, where $g_{r} \in Q^{\prime}$ and $f_{r} \in Q$. Hence if $Q$ is finitely generated and $I R[X]$ contains $Q$, then $Q=Q^{\prime}$.

b. Let $f$ be a homogeneous polynomial in $n+1$ variables with coefficients in $R$. Then $f\left(x, x_{1}, \cdots, x_{n}\right)=0$ if, and only if, $f\left(1, X_{1}, \cdots, X_{n}\right) \in Q$. To see "only if" divide the equation $f\left(x, x_{1}, \cdots, x_{n}\right)=0$ by $x^{t}$, where $t$ is the degree of $f$; to see "if" multiply the equation $f\left(1, x_{1} / x, \cdots, x_{n} / x\right)=0$ by $x^{t}$. Thus if $f\left(x, x_{1}, \cdots, x_{n}\right)=0$, then the coefficients of $f$ lie in every ideal $P$ of $R$ such that $P R[X]$ contains $Q$.

A subset $\left\{z_{1}, \cdots, z_{m}\right\}$ of $R$ is said to be analytically independent provided that every homogeneous $f \in R\left[Z_{1}, \cdots, Z_{m}\right]$ such that $f\left(z_{1}, \cdots, z_{m}\right)=$ 
0 must have its coefficients in the radical of $\left(z_{1}, \cdots, z_{m}\right)$; we shall say (for lack of a better term) that the subset is strongly analytically independent if the coefficients of $f$ must lie in $\left(z_{1}, \cdots, z_{m}\right)$. We rephrase Remark b as follows:

$b^{\prime} . \quad\left\{x, x_{1}, \cdots, x_{n}\right\}$ is: analytically independent if, and only if, for every prime ideal $P$ of $R$ containing $I, P R[X]$ contains $Q$; strongly analytically independent if, and only if, $I R[X]$ contains $Q$.

Let $N=\left(z_{1}, \cdots, z_{m}\right)$. The direct sum of the $(R / N)$-modules $\left\{N^{r} / N^{r+1}: r=0,1,2, \cdots\right\}$ is in the obvious way the homomorphic image of the polynomial ring $(R / N)\left[Z_{1}, \cdots, Z_{m}\right]$. This homomorphism induces on the direct sum the structure of a graded $(R / N)$-algebra called the associated graded ring of $N$; we shall say that the associated graded ring is $(R / N)\left[Z_{1}, \cdots, Z_{m}\right]$ if this homomorphism is an isomorphism. Thus:

c. The following statements are equivalent: $\left\{z_{1}, \cdots, z_{m}\right\}$ is strongly analytically independent; the associated graded ring of $N$ is $(R / N)$ $\left[Z_{1}, \cdots, Z_{m}\right]$; for each $r, N^{r} / N^{r+1}$ is a free $(R / N)$-module of rank equal to the number of monomials of degree $r$ in $m$ variables; any set of $m$ generators of $N$ is strongly analytically independent.

Finally, from the well known relationship between the ideals of $S$ and those of $R[X]$ containing $Q$ we obtain:

d. If $P$ is an ideal of $R$ such that $P R[X]$ contains $Q$, then $P S \cap R=P, S / P S=(R / P)[X]$, and $P S$ is prime if $P$ is prime.

2. Ideals of the principal class. In this section we restrict our attention to Noetherian rings. Recall that an ideal generated by $m$ elements is of height at most $m$, and if equality holds, the ideal is said to be of the principal class. ${ }^{1}$ We shall assume that $h t(I)=$ $n+1$; i.e., $I$ is an ideal of the principal class.

\section{Proposition 1. Radical $Q^{\prime}=$ radical $Q$.}

Proof. Let $P$ be a prime ideal of $R[X]$ minimal over $Q^{\prime}$; we must show that $P$ contains $Q$. Since $Q^{\prime}$ is generated by $n$ elements, $h t(P) \leqq n$. Observe that $x \notin P$. For if $x \in P$, then $x_{i}=x X_{i}-Y_{i} \in P$; $P$ would therefore contain $I$ and so be of height at least $n+1$. Since $Q^{\prime}$ contains $x^{r} Q$ for sufficiently large $r$ (Remark a), it follows that $P$ contains $Q$.

${ }^{1}$ We follow Zariski-Samuel in using the term "height" rather than the older "rank". Recall that for a prime ideal $P, \operatorname{ht}(P)$ is the dimension of $R_{P}$, and for an arbitrary ideal $N, \operatorname{ht}(N)$ is the minimum of the heights of all the primes containing $N$. 
REMARK. Observe that since $Q^{\prime}$ contains $x^{r} Q$ and $x$ lies in no prime minimal over $Q^{\prime}$, the minimal primary components of $Q$ and $Q^{\prime}$ coincide. Hence if $Q^{\prime}$ is unmixed, $Q=Q^{\prime}$. This will be the case if $R$ (and so $R[X]$ ) is a Macaulay ring, for it is not hard to see that $Q^{\prime}$ is of height $n$ (i.e., $Q^{\prime}$ is an ideal of the principal class). If $I$ is prime, then $I R[X]$ is a prime ideal of $R[X]$ containing $Q^{\prime}$ and so contains $Q$; hence $Q=Q^{\prime}$ (Remark a). Moreover $Q=Q^{\prime}$ if $Q^{\prime}$ is a prime ideal (again Remark a). These three instances of " $Q$ ' $=Q$ " are special cases of: $Q=Q^{\prime}$ if, and only if, $I$ is of grade $n+1$ (see $\S 3$ ).

Zariski in his fundamental paper [6] was the first to consider the relationship between the rings $R$ and $S$. There, when his $\left\{x, x_{1}, \cdots, x_{n}\right\}$ is a subset of a system of parameters in a local ring, he makes essential use of the analytic independence of systems of parameters. We now note that this property is an immediate corollary to Proposition 1; in fact somewhat more generally we have:

Corollary 1. Every basis of minimal cardinality for an ideal of the principal class is analytically independent.

Proof. Suppose that $\left\{z_{1}, \cdots, z_{n}\right\}$ generates an ideal of height $n$ in the ring $A$. Let $z$ be an indeterminate over $A$. Then $\left\{z, z_{1}, \cdots, z_{n}\right\}$ generates an $A[z]$-ideal of height $n+1$. Set $R=A[z], z=x$ and $z_{i}=x_{i}$. Given a prime ideal $P$ of $R$ containing $I$, then $P R[X] \supset I R[X] \supset$ $Q^{\prime}$. Therefore $P R[X] \supset Q$ (Proposition 1). It follows that $\left\{z, z_{1}, \cdots, z_{n}\right\}$ is analytically independent (Remark $b^{\prime}$ ). Suppose now that $f$ is a homogeneous polynomial with coefficients in $A$ such that $f\left(z_{1}, \cdots, z_{n}\right)=$ 0 . The coefficients of $f$ must lie in the radical of the $A[z]$-ideal $\left(z, z_{1}, \cdots, z_{n}\right)$. Since $z$ is an indeterminate, it follows that the coefficients of $f$ must lie in the radical of the $A$-ideal $\left(z_{1}, \cdots, z_{n}\right)$ (just set $z=0)$.

For the remainder of this section we restrict our attention to integral domains. Recall that given $R_{1}$ a subring of $R_{2}$, the dimension formula holds between $R_{1}$ and $R_{2}$ if for every prime ideal $M$ of $R_{2}$ : $\mathrm{ht}(M)+\operatorname{tr} \cdot \operatorname{dg} \cdot\left[R_{2} / M: R_{1} / M \cap R_{1}\right]=\mathrm{ht}\left(M \cap R_{1}\right)+\operatorname{tr} \cdot \mathrm{dg} \cdot\left[R_{2}: R_{1}\right] . \quad$ The dimension formula always holds between $R$ and $R[X]$ (see, e.g., [1], Appendix 1).

Corollary 2. The dimension formula holds between $R$ and $S$.

Proof. ${ }^{2}$ Let $M$ be a prime ideal of $S$, let $P=M \cap R$, and let $N$ be the pre-image of $M$ in $R[X]$. Thus $N \cap R=P, M=N / Q$ and $S / M=R[X] / N$. We see from the dimension formula between $R$ and $R[X]$ that $h t(Q)=n$. Since $Q$ is the only prime minimal over $Q^{\prime}$

2) Essentially the same proof works even if $R$ is not an integral domain. 
(Proposition 1), $\operatorname{ht}\left(Q^{\prime}\right)=n$; i.e., $Q^{\prime}$ is an ideal of the principal class. Hence $h t\left(N / Q^{\prime}\right)=h t(N)-h t\left(Q^{\prime}\right)$. It also follows from the fact that $Q$ is the only prime minimal over $Q^{\prime}$ that $h t(N / Q)=h t\left(N / Q^{\prime}\right)$; hence $\operatorname{ht}(M)=\operatorname{ht}(N)-n$. The dimension formula between $R$ and $R[X]$ gives:

$$
\mathrm{ht}(N)+\operatorname{tr} \cdot \mathrm{dg} \cdot[S / M: R / P]=\mathrm{ht}(P)+n .
$$

Substituting $h t(N)-n=h t(M)$ gives the desired result.

Lemma. Let $P$ be a prime ideal of $R$ and let $\Delta$ denote the set of all primes $M$ of $S$ such that $M \cap R=P$. (1) If $x \in P$ but $I \not \subset P$, then $\Delta$ is empty. (2) If $x \notin P$, then $\Delta$ consists of a single $M$ and $S_{M}=R_{P}$. (3) If $I \subset P$, then $P S \in \Delta, \Delta$ is infinite, its elements being in one-to-one correspondence with the prime ideals of $(R / P)[X]$ lying over 0 in $R / P$, and for each $M \in \Delta, S_{M I} \neq R_{P}$.

Proof. (1) If $M \in \Delta$, then $I \subset x S \cap R \subset M \cap R=P$. Hence $\Delta$ must be empty. (2) $P R_{P} \cap S$ is the unique $M$ in $\Delta$. (3) $P R[X]$ is a prime ideal of $R[X]$ containing $Q^{\prime}$; hence $P R[X]$ contains $Q$ (Proposition 1). Now apply Remark $d$.

For the remainder of this section was assume that $R$ is local and $P$ is its maximal ideal. Given $M$ a prime ideal of $S$ such that $M \cap R=$ $P$, let $R^{*}=S_{M}$. We shall call $R^{*}$ a local monoidal transform of $R$ with center $I$. These transforms, in the special case of $R$ and $R / I$ regular, play an important role in the work of Zariski, Abhyankar and Hironaka on the resolution of singularities of algebraic varieties. Abhyankar has noted that in this case $R^{*}$ uniquely determines $I$, except in the trivial case of a principal center. We next point out that the reason for this uniqueness is that in this case $I$ is a prime ideal of the principal class-in geometric language, an irreducible local complete intersection.

CoRollary 3. A nontrivial local monoidal transform with center. a prime ideal of the principal class uniquely determines the center.

Proof. Assume that $I$ is prime, and that $R^{*}$ is also the transform of $R$ with center the prime ideal $I^{\prime}=\left(y, y_{1}, \cdots, y_{m}\right)$ of height $m+1$ : $R^{*}=\left(S^{\prime}\right)_{M^{\prime}}$, where $S^{\prime}=R\left[y_{1} / y, \cdots, y_{m} / y\right]$ and $M^{\prime}$ is a prime of $S^{\prime}$ lying over $P$. By the above lemma, $I S$ is prime, $(I S) \cap R=I$ and $S_{I S} \neq R_{I}$. Since $R^{*}$ is a quotient ring of $S$ and of $S^{\prime}, S_{I S}=\left(R^{*}\right)_{I R^{*}}=$ $\left(S^{\prime}\right)_{N}$, where $N=\left(I R^{*}\right) \cap S^{\prime}$. Consequently $\left(S^{\prime}\right)_{N} \neq R_{N \cap R}=R_{I}$; whence: by the lemma, $I=N \cap R \supset I^{\prime}$. Similarly $I^{\prime} \supset I$. 
REMARK. Even if $I$ and $I^{\prime}$ are not prime, the above argument shows that their radicals are identical. Question: are their minimal primary components necessarily the same? If so, then for $I$ and $I^{\prime}$ unmixed (a condition guaranteed for $R$ a Macaulay ring) we may drop the assumption that $I$ and $I^{\prime}$ be prime. This question reduces to: if $I$ and $I^{\prime}$ are $P$-primary and $R^{*}=S_{P S}=\left(S^{\prime}\right)_{P S^{\prime}}$, is $I=I^{\prime}$ ?

3. $R$-sequences. Given an $R$-module $A$, the sequence $\left\{y_{1} \cdots, y_{m}\right\}$ in $R$ is said to be an $A$-sequence provided that $A \neq y_{1} A+\cdots+y_{m} A$ and for $i=1, \cdots, m, y_{1} \notin Z\left(A / y_{1} A+\cdots y_{i-1} A\right)$. (In general $Z(B)$ denotes the set of zero-divisors on the $R$-module $B$; i.e., $Z(B)=\{r \in R$ : multiplication by $r$ is not a monomorphism on $B\}$. The term " $A$-sequence" is probably due to Auslander and Buchsbaum or perhaps to Serre. They had been studied in several contexts by other writers, especially Nagata (see "distinct systems of parameters" [2] and $\S 25$ of [3]). The corollary to Proposition 2 below has a long history; it was proved by: Macaulay [4] for polynomial rings, Nagata [2] for local "Macaulay rings" and by Rees [7] for Noetherian rings. Rees' proof is an adaption of Macaulay's and actually works for arbitrary commutative rings. The proof given below is new.

Proposition 2. If $\left\{x, x_{1}, \cdots, x_{n}\right\}$ is an $R$-sequence, then $Q=Q^{\prime}$. (Hence if $R$ is an integral domain, then $Q^{\prime}$ is a prime ideal.)

Proof. Consider first the case $n=1$. For $f \in Q, x^{t} f \in Q^{\prime}$ (Remark a); i.e., $x^{t} f=\left(x X_{1}-x_{1}\right) g$, with $g \in R\left\lceil X_{1}\right\rceil$. Since $x_{1}$ is clearly not in $Z\left(R /\left(x^{t}\right)\right)$, every coefficient of $g$ must be divisible by $x^{t}$. Canceling the $x^{t}$ shows that $f$ is a multiple of $x X_{1}-x_{1}$. In the general case we consider the homomorphism $R[X] \rightarrow S$ in two stages: $R[X] \rightarrow S^{\prime}\left[X_{2}, \cdots, X_{n}\right] \rightarrow S$, where $S^{\prime}=R\left[x_{1} / x\right]$. We have already seen that $Y_{1}$ generates the kernel of the first stage. Since $\left(x, x_{1}\right) R\left[X_{1}\right]$ contains the kernel of $R\left[X_{1}\right] \rightarrow S^{\prime}, S^{\prime} / x S^{\prime}=S^{\prime} /\left(x, x_{1}\right) S^{\prime}=\left(R /\left(x, x_{1}\right)\right)\left[X_{1}\right]$ (Remark d). Since the residues of $\left\{x_{2}, \cdots, x_{m}\right\}$ in $R /\left(x, x_{1}\right)$ constitute an $\left(R /\left(x, x_{1}\right)\right)$-sequence, and hence an $\left(R /\left(x, x_{1}\right)\right)\left[X_{1}\right]$-sequence, if follows that $\left\{x, x_{2}, \cdots, x_{n}\right\}$ is an $S^{\prime}$-sequence. By induction on $n,\left\{Y_{2}, \cdots, Y_{n}\right\}$ generates the kernel of the second stage. Hence $Q=\left(Y_{1}, \cdots, Y_{n}\right)$.

Since we have already noted that $\left\{x, x_{1}, \cdots, x_{n}\right\}$ is strongly analytically independent if, and only if, $I R[X]$ contains $Q$, we have as an immediate corollary the following theorem of Rees [7]:

COROLLARY. R-sequences are strongly analytically independent. Equivalently: if an ideal $N$ is generated by an $R$-sequence of length $m$, then the associated graded ring of $N$ is $(R / N)\left[Z_{1}, \cdots, Z_{m}\right]$. 
Now if $A$ is a faithful $R$-module and $\left\{x, x_{1}, \cdots, x_{n}\right\}$ is an $A$-sequence, replacing $R[X]$ by the $R[X]$-module of all "polynomials" with coefficients in $A$ (i.e., $R \mid X] \otimes A$ ) and $S$ by $S \otimes A$ in the above argument gives:

Proposition 2'. The kernel of the homomorphism $R[X] \otimes A \rightarrow$ $S \otimes A$ is $Q^{\prime}(R[X] \otimes A)$.

And since the residues of an $A$-sequence in the ring $R /$ annihilator $(A)$ form an $A$-sequence, we have as a corollary the "strong analytical independence of $A$-sequences"; namely:

Corollary. Let $\left\{z_{1}, \cdots, z_{m}\right\}$ be an $A$-sequence. If $f \in A\left[Z_{1}, \cdots, Z_{m}\right]$ $\left(=R\left[Z_{1}, \cdots, Z_{m}\right] \otimes A\right)$ is homogeneous and $f\left(z_{1}, \cdots, z_{m}\right)=0$, then $f \in\left(z_{1}, \cdots, z_{m}\right) A\left[Z_{1}, \cdots, Z_{m}\right]$. (That is, if $\sum f_{i}\left(z_{1} \cdots, z_{m}\right) a_{i}=0$, where the $f_{i}$ are distinct monomials of the same degree and $a_{i} \in A$, then each $a_{i} \in\left(z_{1}, \cdots, z_{m}\right) A$. $)$

REMARK. In the notation of this corollary let $N$ be the ideal generated by $\left\{z_{1}, \cdots, z_{m}\right\}$. It is now an easy exercise to show that $Z(A / N A)=Z\left(A / N^{r} A\right)($ see [7]); somewhat more generally (but just as easy) is: $Z(A / N A)=Z(A / M A)$, where $M$ is any ideal generated by monomials in $\left\{z_{1}, \cdots, z_{m}\right\}$ having the same radical as $N$. These facts are generalizations of Macaulay's Unmixedness Theorem: In the ring of polynomials over a field every power of an ideal of the principal class is unmixed. For these and other generalizations from the point of view of homological dimension consult Kaplansky's paper: $R$ sequences and homological dimension, Nagoya Journal, Vol. 20 (1962), 195-199.

Henceforth assume that $R$ is a Noetherian ring. Given a finitely generated $R$-module $A$ and an ideal $N$ of $R$ such that $A \neq N A$, then any two $A$-sequences in $N$ which are maximal (with respect to being $A$-sequences in $N$ ) have the same length. (For the details see [7], or for a simplified account [5]) Following Rees we shall call this common integer the grade of $N$ on $A$, denoted $G(N, A)$. For $A=R$, we let grade of $N=G(N)=G(N, R)$. In the following theorem we collect several characterizations of ideals generated by $R$-sequences. Such an ideal is clearly an ideal of the principal class; rings for which the converse is true are called Macaulay rings.

Theorem. Let $N$ be an ideal generated by m elements. Then the following statements are equivalent. (1) $G(N)=m$. (2) $N$ is generated by an $R$-sequence of length $m$. (3) The associated graded ring of $N$ is $(R / N)\left[Z_{1}, \cdots, Z_{m}\right]$. (4) For every nonnegative integer 
$r, N^{r} / N^{r+1}$ is a free $(R / N)$-module of rank equal to the number of monomials of degree $r$ in $m$ variables. (5) Every basis of $N$ consisting of $m$ elements is strongly analytically independent. (6) some basis for $N$ consisting of $m$ elements is strongly analytically independent.

If furthermore $R$ is an integral domain, then each of these six conditions is equivalent to: (7) If $N=\left(z_{1}, \cdots, z_{m}\right)$, with $z_{m} \neq 0$, then the polynomials $\left\{z_{m} Z_{i}-z_{i}: 1 \leqq i<m\right\}$ generate a prime ideal in $R\left[Z_{1}, \cdots, Z_{m-1}\right]$.

Proof. The equivalence of (1) and (2) is proved in the appendix; that of (3), (4), (5) and (6) in Remark c. That (2) implies (6) is the corollary of Proposition 2. It remains to consider "(5) implies (1)". This could be accomplished by localizing judiciously and invoking the local version of the theorem given by Rees [7], but we prefer to avoid this technicality and to present a self-contained proof here.

If $G(N) \neq 0$, then we may assume that $N=\left(z_{1}, \cdots, z_{m}\right)$, where $z_{1}$ is not a divisor of zero (see the appendix). It is easy to see that the residues of $\left\{z_{2}, \cdots, z_{m}\right\}$ are strongly analytically independent in $R /\left(z_{1}\right)$. Induction on $m$ then finishes the proof. Hence it suffices to show that $G(N) \neq 0$, or equivalently that if $z N=0$, then $z=0$. Since $z z_{1}=0$, by strong analytic independence, $z \in I$. Suppose $z=$ $f\left(z_{1}, \cdots, z_{m}\right)$, where $f \in R\left[Z_{1}, \cdots, Z_{m}\right]$ is homogeneous of degree $r$. Let $g=Z_{1} f$. Since $g\left(z_{1}, \cdots, z_{m}\right)=0$, the coefficients of $g$ lie in $N$; hence the coefficients of $f$ lie in $N$. It follows that $z=h\left(z_{1}, \cdots, z_{m}\right)$, where $h$ is homogeneous of degree $r+1$. Thus $z \in M=\cap\left\{N^{r}: r=\right.$ $1,2, \cdots\}$. By the intersection theorem of Krull, $1=u+v$, where $u \in N$ and $v \in$ annihilator $(M)$. Hence $z=z u+z v=0$.

Suppose now that $R$ is an integral domain. We change notation so that $I=N$ and $m=n+1$. The proof of the final statement of the theorem then becomes a question of showing that $Q^{\prime}$ is prime if, and only if, $\left\{x, x_{1}, \cdots, x_{n}\right\}$ is strongly analytically independent. And since the strong analytical independence of $\left\{x, x_{1}, \cdots, x_{n}\right\}$ is equivalent to " $I R[X]$ contains $Q$ " (Remark $b^{\prime}$ ), we are reduced to showing that $Q^{\prime}$ is prime if, and only if, $I R[X]$ contains $Q$. This is an immediate consequence of Remark a.

REMARK. In Corollary 3 of Proposition 1 we were concerned with prime ideals of the principal class. Such an ideal $P$ is generated by an $R$-sequence of length $h t(P)$ (by Corollary 1 to Proposition 1 and the above theorem). Hence if $M$ is an ideal properly containing $P$, $G(M)>h t(P)$. Thus in a given ring we must expect bounds on the heights of prime ideals of the principal class. For example, if $R$ is a nonregular local ring with maximal ideal $M$, then every prime ideal 
of the principal class is of height at most $G(M)-1$. Question: is this upper bound always achieved? If we restrict our attention to local domains, this question reduces, by induction, to: if $G(M)>1$, does $R$ have a principal prime ideal ? The correct answer to this question seems to be unknown. Let us note that the familiar technique of increasing the residue field by the adjunction of indeterminates produces prime ideals of the principal class of maximal height. Suppose that $G(M)=G(I)=n+1$. Let $R^{*}=R[X]_{M R[X]}$ and let $M^{*}$ be the maximal ideal of $R^{*}$. Observe that $\operatorname{dimension}\left(R^{*}\right)=\operatorname{dimension}(R), G\left(M^{*}\right)=$ $G(M)$ and that $Q^{\prime} R^{*}$ is a prime ideal of the principal class of height $n$.

APPENDIX. The material in this section is part of some work done by the author while he was a student of Professor Kaplansky at the University of Chicago; we take the opportunity to record it here. We shall need two elementary facts from commutative algebra available in any standard textbook on the subject, (e.g., [1]). Firstly: an ideal not contained in any one of a finite set of prime ideals is not contained in the set-theoretic union. Secondly the following part of the primary decomposition of 0 in a finitely-generated $R$-module $A$, for Noetherian $R: Z(A)$ is the union of a finite set of prime ideals having no containment relation among them.

Lemma. If the ideal $N$ is contained in the union of a finite set of prime ideals having no containment relation among them, and $(N, y)$ is not, then $(N, y)=(N, z)$, where $z$ lies in none of the primes.

Proof. Let $u+r y \notin \cup\left\{P_{i}\right\}$, where $u \in N$. Clearly if $y \in P_{i}$, then $u \notin P_{i}$. We may assume that $y \notin P_{i}(1 \leqq i \leqq j)$ and that $y \in P_{i}(j<i)$. Select $s \in \cup\left\{P_{i}: i \leqq j\right\}-\cap\left\{P_{i}: j<i\right\}$. Then $z=s u+y$ is as desired.

Theorem. If $G\left(\left(y_{1}, \cdots, y_{n}\right), A\right)=m$, then $\left(y_{1}, \cdots, y_{n}\right)=\left(z_{1}, \cdots, z_{n}\right)$, where $\left\{z_{1}, \cdots, z_{m}\right\}$ is an A-sequence.

Proof. Let $Z(A)=\cup\left\{P_{i}\right\}$ as in the primary decomposition mentioned above. Let $k$ be maximal such that $Z(A)$ contains $\left(y_{1}, \cdots, y_{k}\right)$. Then by the lemma, $\left(y_{1}, \cdots, y_{k+1}\right)=\left(z_{1}, y_{1}, \cdots, y_{k}\right)$, where $z_{1} \notin Z(A)$. Replace $R$ by $R /\left(z_{1}\right), A$ by $A / z_{1} A,\left(y_{1}, \cdots, y_{n}\right)$ by the ideal generated by the residues of $\left\{y_{i}: i \neq k+1\right\}$, and proceed by induction on $m$.

CoRollary (Grade Unmixedness Theorem). If an ideal $N$ is generated by $n$ elements and is of grade $n$, then all the associated primes of $N$ are of grade $n$. 
Proof. By the theorem, $N$ is generated by an $R$-sequence of length $n$. That $R$-sequence is then a maximal $R$-sequence in any associated prime of $N$.

\section{REFERENCES}

1. O. Zariski and P. Samuel, Commutative algebra, (2 vols.), Princeton, 1958 and 1960. 2. M. Nagata, The theory of multiplicity in general local rings, Proceedings of the International Symposium, Tokyo-Nikko, 1955, Scientific Council of Japan, Tokyo, 1959, 191-226.

3. _- Local Rings, Interscience, 1962.

4. F. S. Macaulay, The Algebraic theory of modular systems, Cambridge Math. Tract Number 19, Cambridge University Press, 1916.

5. D. Rees and D. G. Northcott, Extensions and simplifications of the theory of regular local rings, J. London Math. Soc. 32 (1957), 367-374.

6. O. Zariski, Foundations of a general theory of birational, correspondences, Trans. Amer. Math. Soc., 53 (1943), 497-542.

7. D. Rees, The grade of an ideal or module, Proc. Camb. Phil. Soc. 53 (1957), 28-42.

Received January 20, 1966. Supported in part by Purdue Research Foundation (PRF-4294), and in part by the National Science Foundation (AP-4249).

Purdue UnIVERSITY 



\section{PACIFIC JOURNAL OF MATHEMATICS}

\section{EDITORS}

\author{
H. SAMELSON \\ Stanford University \\ Stanford, California \\ J. P. JANS \\ University of Washington \\ Seattle, Washington 98105
}

\section{J. DugundJI}

University of Southern California Los Angeles, California 90007

RICHARD ARENS

University of California

Los Angeles, California 90024

\section{ASSOCIATE EDITORS}
E. F. BECKENBACH
B. H. NeumanN
F. WOLF
K. YOSIDA

\section{SUPPORTING INSTITUTIONS}

\author{
UNIVERSITY OF BRITISH COLUMBIA \\ CALIFORNIA INSTITUTE OF TECHNOLOGY \\ UNIVERSITY OF CALIFORNIA \\ MONTANA STATE UNIVERSITY \\ UNIVERSITY OF NEVADA \\ NEW MEXICO STATE UNIVERSITY \\ OREGON STATE UNIVERSITY \\ UNIVERSITY OF OREGON \\ OSAKA UNIVERSITY \\ UNIVERSITY OF SOUTHERN CALIFORNIA
}

\author{
STANFORD UNIVERSITY \\ UNIVERSITY OF TOKYO \\ UNIVERSITY OF UTAH \\ WASHINGTON STATE UNIVERSITY \\ UNIVERSITY OF WASHINGTON \\ AMERICAN MATHEMATICAL SOCIETY \\ CHEVRON RESEARCH CORPORATION \\ TRW SYSTEMS \\ NAVAL ORDNANCE TEST STATION
}

Mathematical papers intended for publication in the Pacific Journal of Mathematics should be typewritten (double spaced). The first paragraph or two must be capable of being used separately as a synopsis of the entire paper. It should not contain references to the bibliography. Manuscripts may be sent to any one of the four editors. All other communications to the editors should be addressed to the managing editor, Richard Arens at the University of California, Los Angeles, California 90024 .

50 reprints per author of each article are furnished free of charge; additional copies may be obtained at cost in multiples of 50 .

The Pacific Journal of Mathematics is published monthly. Effective with Volume 16 the price per volume (3 numbers) is $\$ 8.00$; single issues, $\$ 3.00$. Special price for current issues to individual faculty members of supporting institutions and to individual members of the American Mathematical Society: $\$ 4.00$ per volume; single issues $\$ 1.50$. Back numbers are available.

Subscriptions, orders for back numbers, and changes of address should be sent to Pacific Journal of Mathematics, 103 Highland Boulevard, Berkeley 8, California.

Printed at Kokusai Bunken Insatsusha (International Academic Printing Co., Ltd.), No. 6, 2-chome, Fujimi-cho, Chiyoda-ku, Tokyo, Japan.

PUBLISHED BY PACIFIC JOURNAL OF MATHEMATICS, A NON-PROFIT CORPORATION

The Supporting Institutions listed above contribute to the cost of publication of this Journal, but they are not owners or publishers and have no responsibility for its content or policies. 


\section{Pacific Journal of Mathematics \\ Vol. 20, No. $2 \quad$ October, 1967}

Edward Dewey Davis, Ideals of the principal class, $R$-sequences and a certain monoidal transformation ............................. 197

Richard Mansfield Dudley, Sub-stationary processes ................ 207

Newton Seymour Hawley and M. Schiffer, Riemann surfaces which are doubles of plane domains ......................... 217

Barry E. Johnson, Continuity of transformations which leave invariant certain translation invariant subspaces ................... 223

John Eldon Mack and Donald Glen Johnson, The Dedekind completion of

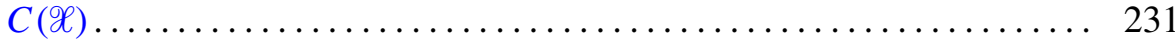

K. K. Mathur and R. B. Saxena, On the convergence of quasi-Hermite-Fejér interpolation................................... 245

H. D. Miller, Generalization of a theorem of Marcinkiewicz............ 261

Joseph Baruch Muskat, Reciprocity and Jacobi sums ............... 275

Stelios A. Negrepontis, On a theorem by Hoffman and Ramsay .......... 281

Paul Adrian Nickel, A note on principal functions and multiply-valent canonical mappings .............................. 283

Robert Charles Thompson, On a class of matrix equations ............. 289

David Morris Topping, Asymptoticity and semimodularity in projection lattices ........................................ 317

James Ramsey Webb, A Hellinger integral representation for bounded linear functionals

Joel John Westman, Locally trivial $C^{r}$ groupoids and their representations...

Hung-Hsi Wu, Holonomy groups of indefinite metrics 